PROCEEDINGS OF THE

AMERICAN MATHEMATICAL SOCIETY

Volume 130, Number 6, Pages 1805-1808

S 0002-9939(01)06220-7

Article electronically published on December 20, 2001

\title{
A RICCI-SEMI-SYMMETRIC HYPERSURFACE OF EUCLIDEAN SPACE WHICH IS NOT SEMI-SYMMETRIC
}

\author{
BAHAA E. ABDALLA AND FRANKI DILLEN
}

(Communicated by Wolfgang Ziller)

Abstract. We construct the first explicit example of a Ricci-semi-symmetric hypersurface of Euclidean space which is not semi-symmetric.

\section{Preliminaries and introduction}

Let $\left(M^{n}, g\right)$ be a Riemannian manifold of class $C^{\infty}$. Let $R$, defined by

$$
R(X, Y) Z=\left[\nabla_{X}, \nabla_{Y}\right] Z-\nabla_{[X, Y]} Z,
$$

denote the Riemann-Christoffel curvature tensor of $M^{n}$ and let $S$, defined by

$$
S(Y, Z)=\operatorname{trace}\{X \rightarrow R(X, Y) Z\},
$$

denote the Ricci tensor of $M^{n}$. For any $(0, k)$-tensor field $T$ on $M^{n}$, we define a $(0, k+2)$-tensor field $R \cdot T$ by

$$
\begin{gathered}
(R \cdot T)\left(X_{1}, \ldots, X_{k} ; X, Y\right)=-T\left(R(X, Y) X_{1}, X_{2}, \ldots, X_{k}\right) \\
-T\left(X_{1}, R(X, Y) X_{2}, \ldots, X_{k}\right)-\cdots-T\left(X_{1}, \ldots, X_{k-1}, R(X, Y) X_{k}\right) .
\end{gathered}
$$

A Riemannian manifold $\left(M^{n}, g\right)$ is said to be semi-symmetric if

$$
R \cdot R=0
$$

on $M^{n}$. It is well known that the class of semi-symmetric manifolds includes the set of locally symmetric manifolds $(\nabla R=0)$ as a proper subset. A Riemannian manifold $\left(M^{n}, g\right)$ is said to be Ricci-semi-symmetric if

$$
R \cdot S=0
$$

The class of Ricci-semi-symmetric manifolds includes the set of Ricci-symmetric manifolds $(\nabla S=0)$ as a proper subset. Evidently, condition (1.1) implies condition (1.2). The converse is in general not true. However, under certain conditions (for instance if $n=3$ ), (1.1) and (1.2) are equivalent; see [3] for an account of results in this direction.

A long-standing open problem is the following: are conditions (1.1) and (1.2) equivalent for hypersurfaces in Euclidean space? This problem is known as Ryan's

Received by the editors July 28, 2000 and, in revised form, November 28, 2000.

1991 Mathematics Subject Classification. Primary 53B25.

Key words and phrases. Semi-symmetric, Ricci-semi-symmetric, hypersurface.

The second author is a Postdoctoral Research Fellow of the Fund for Scientific ResearchFlanders. 
problem, although P. J. Ryan never stated this problem explicitly for hypersurfaces in Euclidean space. In his papers [7], [8], Ryan studies the relation between the two conditions, proving for instance that conditions (1.1) and (1.2) are equivalent for hypersurfaces in spheres and hyperbolic spaces and for hypersurfaces of Euclidean space with nonnegative scalar curvature. Another interesting result in this direction is proved in [5]: conditions (1.1) and (1.2) are equivalent for complete hypersurfaces of Euclidean space. Also it is proved in 2 that conditions (1.1) and (1.2) are equivalent for hypersurfaces of 5-dimensional semi-Riemannian space of constant curvature. Finally, F. Defever shows in [1] the existence of a 5dimensional hypersurface of Euclidean space that is Ricci-semi-symmetric but not semi-symmetric. Defever's result is an existence result: no explicit examples are given. In the present paper we give for all dimensions $n>4$ an easy and explicit example of a hypersurface in Euclidean space $\mathbb{E}^{n+1}$ that is Ricci-semi-symmetric but not semi-symmetric.

\section{The EXAmple}

Let $M^{n}$ be a hypersurface of the Euclidean space $\mathbb{E}^{n+1}$. Denote by $\nabla$ and $D$ the Levi-Civita connection of $M^{n}$ and $\mathbb{E}^{n+1}$ respectively. For any vector fields $X, Y$ tangent to $M^{n}$, the formula of Gauss is given by

$$
D_{X} Y=\nabla_{X} Y+h(X, Y) \xi
$$

where $h$ is the scalar-valued second fundamental form, and $\xi$ a unit normal vector. The formula of Weingarten is given by

$$
D_{X} \xi=-A(X)
$$

where $A$ is the shape operator of $\xi$. The shape operator is related to the second fundamental form $h$ by

$$
g(A(X), Y)=h(X, Y)
$$

where $g$ is the induced metric on $M^{n}$. Then the equation of Gauss is

$$
R(X, Y) Z=g(A(Y), Z) A(X)-g(A(X), Z) A(Y) .
$$

Now, we take any point $p \in M$ and an orthonormal basis $\left\{e_{1}, \ldots, e_{n}\right\}$ at $p$ which diagonalizes the shape operator $A$ with principal curvatures $\lambda_{i}(i=1, \ldots, n)$. With respect to this frame, the only nonzero components of the Riemann-Christoffel curvature tensor are

$$
R_{i j j i}=\lambda_{i} \lambda_{j}, \quad i \neq j, 1 \leq i, j \leq n,
$$

and the Ricci tensor is diagonal with eigenvalues

$$
S_{i i}=-\lambda_{i}\left(\sum_{i \neq j} \lambda_{j}\right)
$$

Using the equation of Gauss, the set of equations for $R \cdot R=0$ amounts to

$$
\lambda_{i} \lambda_{j} \lambda_{k}\left(\lambda_{i}-\lambda_{j}\right)=0, \quad i \neq j, j \neq k, k \neq i, \quad 1 \leq i, j \leq n .
$$

The set of equations for $R \cdot S=0$ amounts to

$$
\lambda_{i} \lambda_{j}\left(\lambda_{i}-\lambda_{j}\right)\left(\sum_{k \neq i, k \neq j} \lambda_{k}\right)=0, \quad i \neq j, \quad 1 \leq i, j, k \leq n .
$$


We remark that any solution of (2.3) is indeed a solution of (2.4). We remark that equations (2.3) and (2.4) appeared first in [6] and [7] respectively.

In 11, Defever proved the existence of a hypersurface $M^{5}$ of $\mathbb{E}^{6}$ which has principal curvatures $(0, b, b,-b,-b)$ at every point $p \in M^{5}$, for some function $b$ on $M^{5}$. In this section we give an easy explicit example of such a hypersurface. It is clear that a hypersurface with principal curvatures $(0, b, b,-b,-b)$ at every point is Ricci-semi-symmetric but not semi-symmetric, assuming $b \neq 0$. More general, a hypersurface with principal curvatures $(0, b, \ldots, b,-b, \ldots,-b)$, where $b$ and $-b$ have the same multiplicity, is Ricci-semi-symmetric but not semi-symmetric.

Let $n>1$ and $S^{n}=\left\{p \in \mathbb{R}^{n+1}\right.$ such that $\left.|p|=1\right\}$ be the standard unit sphere. First consider

$$
M^{2 n}=S_{1}^{n} \times S_{2}^{n}=\left\{(p, q) \in \mathbb{R}^{2 n+2}=\mathbb{R}^{n+1} \times \mathbb{R}^{n+1} \text { such that }|p|=|q|=1\right\} .
$$

Next we take the cone $\mathcal{C}^{2 n+1}$ on $M^{2 n}$, with top at the origin, such that a parametrization is given by

$$
\mathcal{C}^{2 n+1}=\left\{(t p, t q) \in \mathbb{R}^{2 n+2} \text { such that }|p|=|q|=1, t \in \mathbb{R}, t>0\right\} .
$$

It can be easily seen that the unit normal of $\mathcal{C}^{2 n+1}$ is given by

$$
\xi=\frac{1}{\sqrt{2}}(-p, q) \text {. }
$$

To evaluate the shape operator of $\mathcal{C}^{2 n+1}$, let $u$ and $v$ be parameters in $S_{1}^{n}$ and $S_{2}^{n}$, respectively. Then we have that

$$
\partial_{u}=\left(t p_{u}, 0\right), \quad \partial_{v}=\left(0, t q_{v}\right), \quad \partial_{t}=(p, q) .
$$

We also have that

$$
D_{\partial_{u}} \xi=\frac{-1}{\sqrt{2}}\left(p_{u}, 0\right), \quad D_{\partial_{v}} \xi=\frac{1}{\sqrt{2}}\left(0, q_{v}\right), \quad D_{\partial_{t}} \xi=0 .
$$

Now using (2.2), we find that

$$
A\left(\partial_{t}\right)=0, \quad A\left(\partial_{u}\right)=\frac{1}{\sqrt{2} t} \partial_{u}, \quad A\left(\partial_{v}\right)=\frac{-1}{\sqrt{2} t} \partial_{v} .
$$

Hence the principal curvatures of $\mathcal{C}^{2 n+1}$ are given by $\left(0, \frac{1}{\sqrt{2} t}, \ldots, \frac{1}{\sqrt{2} t}, \frac{-1}{\sqrt{2} t}, \ldots, \frac{-1}{\sqrt{2} t}\right)$.

Thus we have constructed a hypersurface of every even-dimensional Euclidean space of dimension at least 6 which is Ricci-semi-symmetric but not semi-symmetric. We can remark that we can obtain a hypersurface of every odd-dimensional Euclidean space $\mathbb{R}^{2 n+1}$ of dimension at least 7 which is Ricci-semi-symmetric but not semi-symmetric by considering the product of a cone in $\mathbb{R}^{2 n}$ with a line $\mathbb{R}$.

Recently, a class of non-semi-symmetric Ricci-semi-symmetric hypersurfaces of semi-Euclidean space forms was described in [4].

\section{ADDED IN PROOF}

Recently we were informed by V. A. Mirzoyan that he has obtained an intrinsic classification of Ricci-semi-symmetric hypersurfaces of Euclidean space.

[Mirzoyan, V. A., Classification of Ric-semiparallel hypersurfaces in Euclidean spaces, Sb. Math. 191 (2000), no. 9, 1323-1338.] 


\section{REFERENCES}

[1] F. Defever, Solution of a problem of P.J. Ryan, preprint 1999.

[2] F. Defever, R. Deszcz, Z. Senturk, L. Verstraelen, S. Yaprak, P.J. Ryan's problem in semiEuclidean spaces, Glasgow Math. J., 41 (1999), pp. 271-281. MR 2000d:53054

[3] R. Deszcz, On the equivalence of Ricci-semisymmetry and semisymmetry, Department of Mathematics, Agricultural University Wroclaw, Ser. A, Theory and Methods, Preprint No. 64, Wroclaw 1998.

[4] R. Deszcz and M. Glogowska, Examples of nonsemisymmetric Ricci-semisymmetric hypersurfaces, Department of Mathematics, Agricultural University Wroclaw, Ser. A, Theory and Methods, Preprint No. 79, Wroclaw 2000.

[5] Y. Matsuyama, Complete hypersurfaces with $R \cdot S=0$ in $\mathbb{E}^{n+1}$, Proc. Amer. Math. Soc. 88 (1983), pp. 119-123. MR 84h:53023

[6] K. Nomizu, On hypersurfaces satisfying a certain condition on the curvature tensor, Tôhoku Math. J. 20 (1968), pp. 46-59. MR 37:2138

[7] P. J. Ryan, Hypersurfaces with parallel Ricci tensor, Osaka J. Math. 8 (1971), pp. 251-259. MR 45:5918

[8] - A class of complex hypersurfaces, Colloq. Math. 26 (1972), pp. 175-182. MR 51:1689

Department of Mathematics, College of Science, King Fahd University of Petroleum and Minerals, Box 859, Dhahran 31261, Saudi Arabia

E-mail address: b_abdalla@hotmail.com

Current address: Prince Sultan Private College, Riyadh 11586, P.O. Box 66833, Kingdom of Saudi Arabia

Departement Wiskunde, Katholieke Universiteit Leuven, Celestijnenlaan 200B, B3001 Leuven, Belgium

E-mail address: Franki.Dillen@wis.kuleuven.ac.be 